\title{
Tailoring the magnetization states in 2-D arrays of multiresponse ferromagnetic nanomagnets
}

\author{
M Abuín ${ }^{1,2}$, M Maicas ${ }^{3}$, M Ángel García ${ }^{4,5}, \mathbf{L}$ Pérez $^{1,5}, \mathbf{A}$ \\ Mascaraque ${ }^{1,5}$ \\ ${ }^{1}$ Dpto. de Física de Materiales, Universidad Complutense de Madrid, 28040 Madrid, \\ Spain \\ 2 CEI Campus Moncloa, UCM-UPM, 28040 Madrid, Spain \\ ${ }^{3}$ Institute for Systems based on Optoelectronics and Microtechnology (ISOM), \\ Universidad Politécnica de Madrid, 28040 Madrid, Spain \\ ${ }^{4}$ Instituto de Cerámica y Vidrio, Consejo Superior de Investigaciones Científicas, \\ 28049 Madrid, Spain \\ ${ }^{5}$ Instituto de Magnetismo Aplicado, Universidad Complutense de Madrid-ADIF, \\ 28230 Las Rozas, Spain \\ E-mail: a.mascaraque@ucm.es
}

\begin{abstract}
.
We have fabricated $\mathrm{Fe}_{52-54} \mathrm{Co}_{46-48}$ nanomagnet arrays as a function of several geometrical parameters like the spacing between nanostructures, the aspect ratio and the layers thicknesses. The nanomagnets consist in two magnetic layers, separated by a non magnetic interlayer, that interact through magnetostatic coupling. They present a multiresponse hysteresis loops with two different switching fields. We have performed micromagnetic simulations to discern the role play by the different interactions. The spacing in the array strongly modifies the saturating field along the short axis and the magnetization reversal mechanisms from coherent rotation to domain wall nucleation. A small asymmetry between the two magnetic layers favors a magnetization reversal mechanism along the long axis with two different switching fields. These fields can be tailored through the thickness of the layers or the inter-element spacing in the array. In trilayers with the same magnetic layer thicknesses, the asymmetry can be induced by growing the two magnetic layers with a different anisotropy. The well-defined reversal fields make these nanomagnets potentially useful for magnetic tagging.
\end{abstract}


Tailoring the magnetization states in 2-D arrays of multiresponse ferromagnetic nanomagnets2

\section{Introduction}

The study of interacting magnetic nanoelements is currently one of the most active and innovative area in the magnetism roadmap [1]. Nanomagnets have potential applications in a wide range of fields like 3-D memories [2], magnetic logic devices [3, 4], artificial ferroic systems [5], high frequency emission devices [6] and biomedical applications [7, 8]. Recent developments have introduced nanoelements with dipolar coupling, allowing the construction of multibit switching structures that can be exploited for the fabrication of magnetic tags [9]. In well-ordered arrays of interacting nanomagnets, the shape anisotropy originated from the elements geometry and the inter-element spacing strongly condition the magnetic behaviour.

Within this field, multilayered magnetic nanomagnets are very challenging as they exhibit a magnetostatic coupling between the different magnetic layers that compete with the coupling between neighbour structures in the array. Thus, a milestone for the use of nanomagnets will be the ability to tune their behaviour by tailoring their magnetic coupling in order to optimize them for specific applications. One of the most straightforward methods is to explode proximity effects: nanomagnets in direct contact with other type of magnetic materials alter their behavior due to proximity effects as exchange bias, exchange coupling, etc.. Even if there is no direct contact, nanomagnets close enough can interact through dipolar interactions altering their response to an external magnetic field.

In this paper we analyze a system formed by nanomagnets consisting on trilayers $(\mathrm{FeCo} / \mathrm{Au} / \mathrm{FeCo})$ ordered in two dimensional (2-D) arrays. By growing arrays with different spacings, it is possible to tailor the behavior of the system ranging from single to multiresponse hysteresis loops. Our calculations shown that the two different and well defined switching fields are the result of a competition of the magnetic interactions between the two ferromagnetic layers of a trilayer due to the dipolar interactions between them. Moreover, by comparing experiments and simulation we identify the domains formation and magnetization reversal processes depending on the geometry of the system that leads to the modification of the magnetic properties. These magnetic signatures are valuable for the fabrication of magnetic tags with high magnetic sensitivity.

\section{Methods}

\subsection{Experimental methods}

We have grown $\mathrm{Fe}_{52-54} \mathrm{Co}_{46-48} / \mathrm{Au} / \mathrm{Fe}_{52-54} \mathrm{Co}_{46-48} \quad(\mathrm{FeCo}(1) / \mathrm{Au} / \mathrm{FeCo}(2)$ in the following) trilayer nanomagnets on top of a Silicon wafer using both DC and a Pulsed DC magnetron sputtering sources to induce the different anisotropies in the two magnetic layers as described in previous work [10]. Final nanomagnets are capped with $\mathrm{Au}$ (5 $\mathrm{nm})$ to prevent oxidation.

2-D arrays of nanomagnets were grown using a combination of magnetron sputtering and electron-beam lithography, which is ideal for fabricating large-area 
arrays of identical nanomagnets. The patterns were created with an electron-beam lithography system based on a field-emission type filament (CRESTEC CABL-9500C). The dimensions of the nanomagnets were $1000 \mathrm{~nm}$ of length and $100 \mathrm{~nm}$ of width. The arrays were grown on Si substrates with $5 \mathrm{~nm}$ buffer and capping layers of $\mathrm{Au}$ to prevent oxidation. The different separations of the arrays are described in the text.

Magnetization curves were recorded by means of a Magneto-optical Kerr effect system working in longitudinal configuration and p polarization using a $633 \mathrm{~nm}$ laser in diffraction mode to minimize the substrate contribution [11]. The data presented correspond to the $n=1$ spot. Atomic Force Microscopy (AFM) images were measured with a Nanotec microscope in tapping mode. The software to control and analyze the AFM was the WSxM package [12]. The Scanning Electron Microscope used was a CRESTEC model CABL-9500C.

\subsection{Micromagnetic simulations}

Simulations were done with the LLG Micromagnetics simulator [13]. Sample dimensions were $1000 \mathrm{~nm} \times 100 \mathrm{~nm} \times 20 \mathrm{~nm}$ for single layer nanomagnets and $1000 \mathrm{~nm}$ $\times 100 \mathrm{~nm}$ with thicknesses $20 \mathrm{~nm} / 10 \mathrm{~nm}$ (non-magnetic)/ $20 \mathrm{~nm}$ for magnetic trilayer nanomagnets with a non-magnetic spacer. No thermal effects were considered. Saturation magnetization for FeCo was $\mathrm{M}_{s}=1900 \mathrm{emu} / \mathrm{cm}^{3}$. The effect of crystalline anisotropy was found to be very small compared to shape anisotropy. Results shown were obtained neglecting crystalline anisotropy unless specifically stated. A value of $\mathrm{A}_{e x}=3.510^{-6} \mathrm{erg} / \mathrm{cm}$ was used as exchange constant. This value is much higher than that for pure iron [14] and it has been obtained from light scattering studies [15]. With these parameters we obtain a $4 \mathrm{~nm}$ exchange length so cell dimensions for each layer were taken as $4 \mathrm{~nm} \times 4 \mathrm{~nm} \times \mathrm{t}$ being t the layer thickness. We considered only one single cell along the thickness for each layer in monolayer and bilayer structures as changes in magnetization orientation along the thickness are very small. Convergence criteria (minimum change in any director cosine) was $2 \cdot 10^{-4}$. X-axis and y-axis were assumed as the long and short axis respectively. Calculations have been made considering a small deviation in the applied field $\left(0.57^{\circ}\right)$ from the corresponding axis (x-axis for the long axis loops and y-axis for the short axis loops) as this is closer to experimental conditions where it is difficult to align the applied field with a higher precision.

Parallel processing techniques help in reducing simulation time, although they do not solve the rapidly increasing memory demand in large arrays [16]. Our approach to the study of magnetization processes in large arrays of magnetic nanoelements consist in the use Periodic Boundary Conditions (PBC). These calculations take into account the magnetostatic coupling between nanomagnets in the array under the assumption that all nanostructures behave the same way. Such kind of calculations is not absolutely convergent in three dimensions (3-D), but they have proved to be successful in the study of magnetization processes in large arrays of 2-D magnetic nanostructures [17]. 
Tailoring the magnetization states in 2-D arrays of multiresponse ferromagnetic nanomagnets4

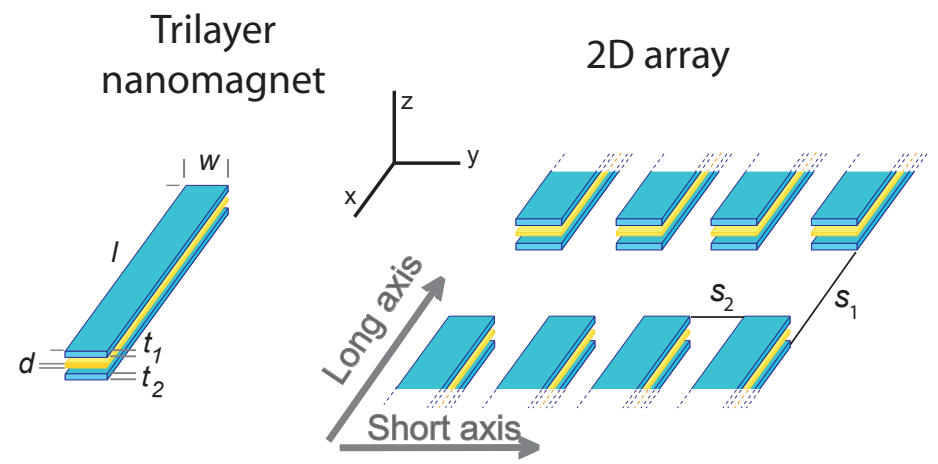

Figure 1. Scheme of the systems studied: isolated trilayer nanomagnet and 2-D array of trilayer nanomagnets. The definition of the different geometrical dimensions used is shown.

\section{Results and Discussions}

\subsection{Fabricated 2-D arrays}

We have studied the magnetic behaviour for fabricated 2-D arrays of $\mathrm{FeCo}(1) / \mathrm{Au} / \mathrm{FeCo}(2)$ trilayer nanomagnets with a constant spacing of $s_{1}=1000 \mathrm{~nm}$ along the long axis and different spacing $\left(s_{2}\right)$ along the short axis. Together with the length $l=1000 \mathrm{~nm}$ and the width $w=100 \mathrm{~nm}$, the relevant dimensions of these nanostructures are the thickness of the two magnetic layers $\left(t_{1}\right.$ and $\left.t_{2}\right)$ and the non-magnetic separation $(d)$. The description of the geometrical parameters is shown in Fig. 1. With $t_{1}=t_{2}=20 \mathrm{~nm}$ of FeCo magnetic layers we still have in-plane magnetization but with enough magnetic material suitable for magnetic applications. The samples have been grown inducing two different magnetocrystalline anisotropies in the two magnetic layers $\left(K_{1}=1.1 \cdot 10^{5} \mathrm{erg} / \mathrm{cm}^{3}\right.$ and $K_{2}=6.7 \cdot 10^{4} \mathrm{erg} / \mathrm{cm}^{3}$ ) using growing parameters as described in [10].

A 2-D array of nanomagnets can be assembled combining thin film growing techniques with nanolithography so the array can cover a large surface area. The e-beam lithography allow us to easily change the spacing parameter $\mathrm{s}_{2}$, making the device technologically feasible. Panel (a) of Fig. 2 shows a sample with a $7 \times 7 \mathrm{~mm}^{2}$ array of nanomagnets with $s_{1}=950 \pm 50 \mathrm{~nm}, s_{2}=150 \pm 5 \mathrm{~nm}$ and $d=10 \pm 2 \mathrm{~nm}$. We present several SEM images that provide information at different scales: from the single nanomagnet to the overall order in the array. Two different samples corresponding to two different $s_{2}$ values are displayed; from a separation of only some tens of $\mathrm{nm}$ (25 nm $\pm 5 \mathrm{~nm}$ ) (panel (d)) up to hundreds of $\mathrm{nm}(538 \mathrm{~nm} \pm 50 \mathrm{~nm}$ ) (panel (c)). The quality of the array in terms of regularity and homogeneity, both in shape, sizes and separation distances is extremely high, regardless the values of the isolated nanomagnet dimensions or their separations in the array.

To check the thicknesses of the layers, we have obtained AFM images (Fig. 3). We have plotted a line profile of the image, that confirms the experimental calibration for $t_{1}$, 
(a)

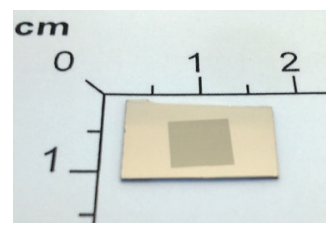

(b)

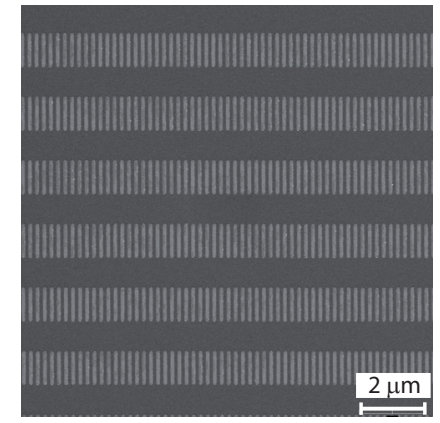

(d)

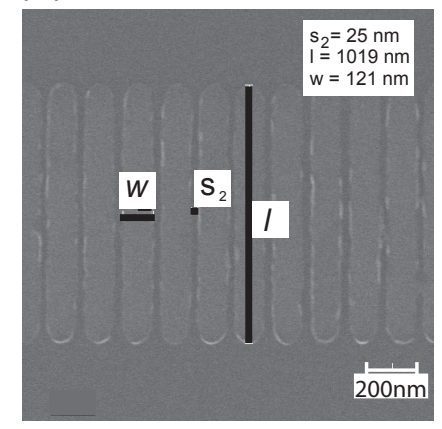

(c)

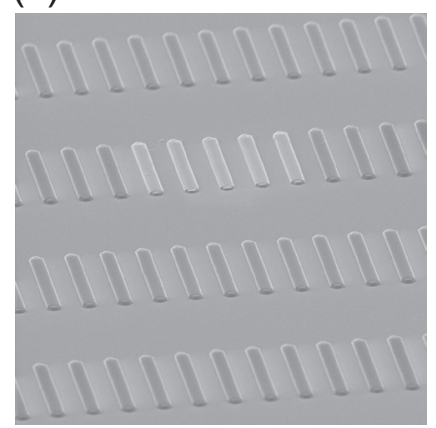

(e)

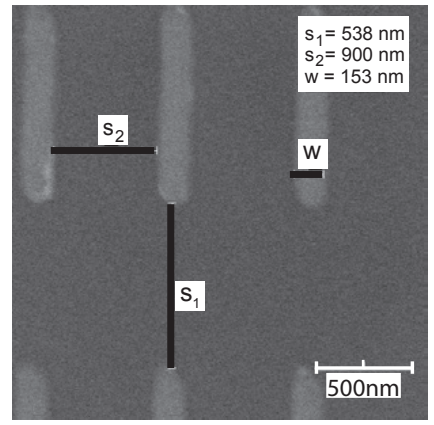

Figure 2. (a) Optical image of FeCo nanomagnets array on $\mathrm{Si}(100)$ substrate. The darkest area $\left(7 \times 7 \mathrm{~mm}^{2}\right)$ corresponds to the square lithographed by e-beam lithography. SEM images of different arrays showing the long range order: (b) $17 \times 17$ $\mu \mathrm{m}^{2}$ of array and (c) SEM image perspective of an array before lift-off. SEM images of two samples with different spacing along $\mathrm{x}$ and y axis with $s_{2}=$ (d) $25 \pm 5 \mathrm{~nm}$ (e) 538 $\pm 15 \mathrm{~nm}$. Each nanomagnet is formed as an stacking of several layers which in the case of these images are: $\mathrm{Au}(5 \mathrm{~nm}) / \mathrm{FeCo}(1)(20 \mathrm{~nm}) / \mathrm{Au}(10 \mathrm{~nm}) / \mathrm{FeCo}(2)(20 \mathrm{~nm}) / \mathrm{Au}(5 \mathrm{~nm})$.

$t_{2}, d$, the buffer and the capping layers of Au. The total thickness of the nanomagnets is $60 \pm 5 \mathrm{~nm}$. As it can be seen down to this magnification, the size and shape of the sample are regular and homogeneous. No impurities are observed. Although the lateral spacing $s_{2}$ is much smaller than the width $w$, the nanomagnets are geometrically isolated from their neighbours, with sharp and straight borders.

The collective magnetic behavior of the arrays is determined by the switching behavior of the individual nanomagnets and by magnetostatic interactions between them. The experimental hysteresis loops of the fabricated samples are represented in Fig. 4. This figure displays the loops along both the short and the long axis for five selected 2-D arrays with the same geometrical parameters but with different $s_{2}$ spacings. 
Tailoring the magnetization states in 2-D arrays of multiresponse ferromagnetic nanomagnets6

(a)

(b)
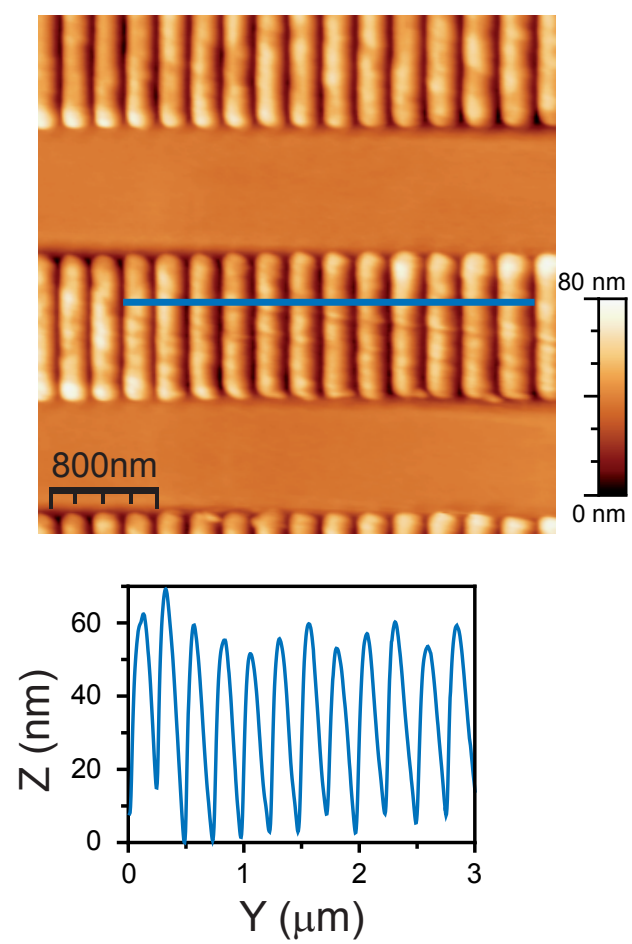

Figure 3. (a) AFM characterization. (a) Topological AFM image in array of FeCo trilayer nanomagnets. The image shows the nanomagnets distribution along $4 \times 4$ $\mu \mathrm{m}^{2}$ of $\mathrm{Si}(100)$. (b) Depth profile measured from twelve nanomagnets along y-axis. The dashed line in the graph correspond to the nominal height of nanomagnets grown in this samples.

Along the short axis magnetization reversal is not abrupt (Fig. 4b). However, along the long axis (Fig. 4a), the samples exhibit a multiresponse hysteresis loops with two different switching fields. For the three larger spacing $\left(s_{2}=180-550 \mathrm{~nm}\right)$ these switching fields are well separated and clearly split. The two jumps can be associated to the reversal of magnetization in each magnetic layer. The first switching field corresponds to the reversal of one single layer on each ribbon and the second to the reversal of the remaining layer. When the spacing becomes larger the ribbons tend to behave as isolated. All of them have the same geometry so they tend to behave in a similar way.

When the $s_{2}$ spacing is reduced the two switching fields come closer. At $s_{2}=40 \pm 5$ $\mathrm{nm}$ the difference is reduced but they are still differentiated. At the lowest spacing $s_{2}=25 \pm 5 \mathrm{~nm}$ only one single switching can be observed. To understand this behavior, it is necessary to take into account the shape anisotropy of the ribbons and the lateral coupling between them. The effect of the shape anisotropy is to produce a strong easy axis along the $\mathrm{x}$ direction and so magnetization is only stable along the two antiparallel orientations. The lateral coupling reduces this anisotropy as the spacing $s_{2}$ becomes smaller. However, for the spacing considered $\left(s_{2}=25-550 \mathrm{~nm}\right)$, the shape anisotropy remains predominant as abrupt magnetization jumps are observed in all cases. 
Tailoring the magnetization states in 2-D arrays of multiresponse ferromagnetic nanomagnets7
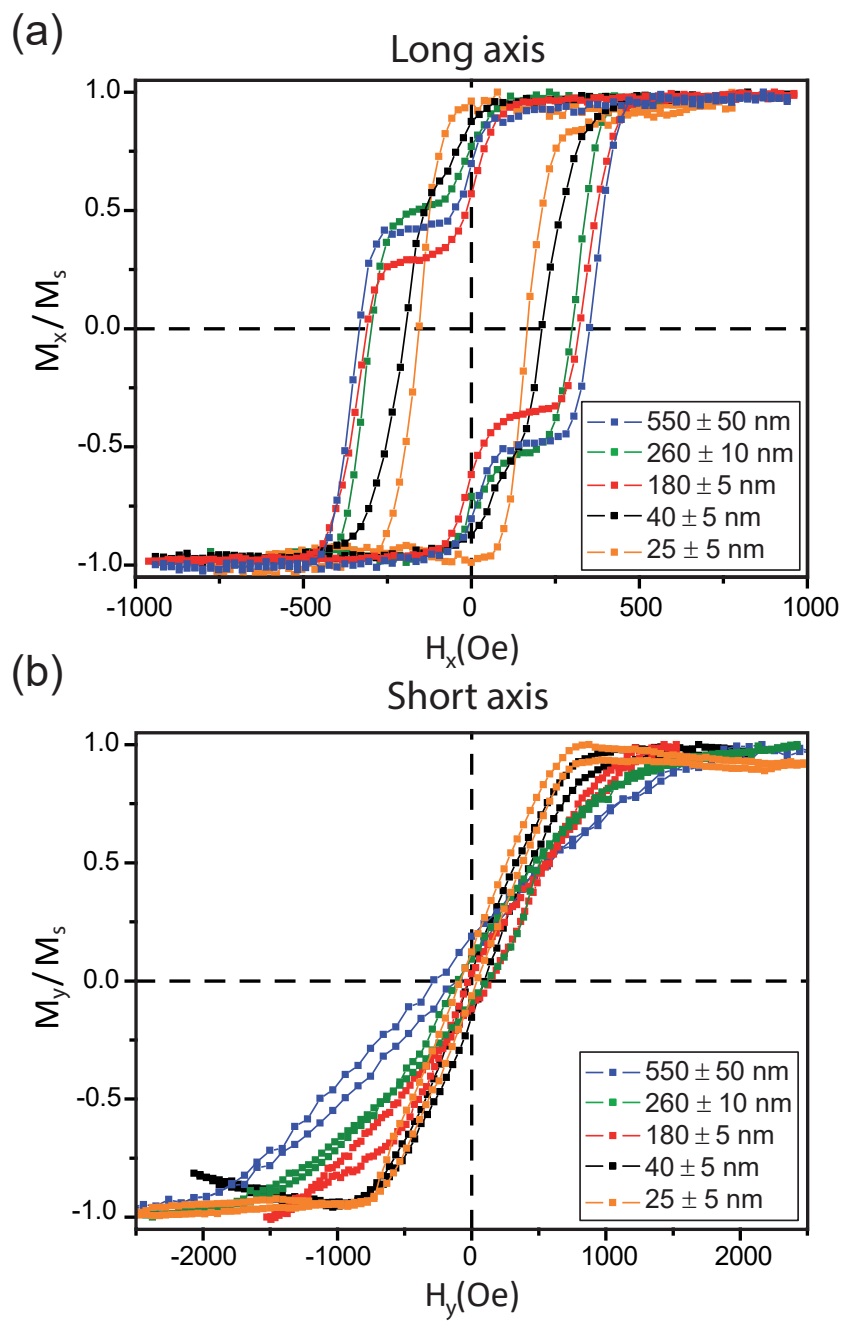

Figure 4. D-MOKE hysteresis loops of three selected different arrays of FeCo trilayer nanomagnets along the long (a) and short (b) axis. Each sample has a different spacing along $\mathrm{y}$-axis $\left(s_{2}\right)$. The blue, red and black hysteresis loops correspond the arrays shown in Fig. 2.

A reduction in the lateral coupling between ribbons makes the demagnetizing field along the $x$ axis increase. The demagnetizing field contributes, as well as the external field, to magnetization reversal. The effect of this demagnetizing field is more important for lower spacing. At low spacing, once magnetization switching begins in some ribbons the demagnetizing field is reduced and so the external field must be increased to continue with the reversal process in other ribbons. So the global switching does not take place in all ribbons at the same time and the hysteresis loop is the result of overlapping hysteresis loops from all ribbons.

Concerning the height of the jumps we would like to point out that they are not representative of the magnetization of each layer since the intensity of the light reflected by the upper layer is larger than that corresponding to the bottom one.

In Fig. 5 we plot the experimental switching fields as a function of the lateral 
Tailoring the magnetization states in 2-D arrays of multiresponse ferromagnetic nanomagnets8
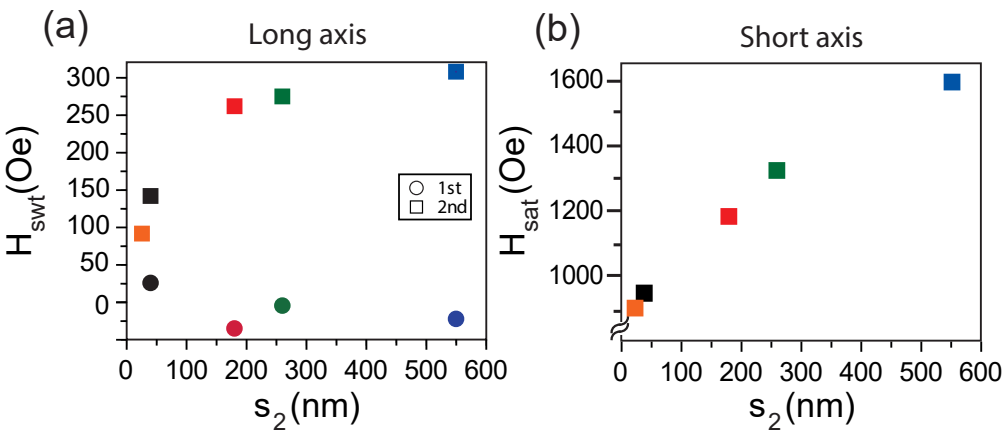

Figure 5. Experimental values for (a) the switching magnetic fields along the long axis necessary for magnetization reversal and (b) saturation field as a function of the spacing between adjacent nanomagnets. The circles mark the first jump, while the squares indicate the second one.

spacing $s_{2}$. Despite the first switching field exhibit no clear dependence on $s_{2}$ taking always values close to zero, the second switching field can be tuned by $s_{2}$, being the larger the spacing the larger the switching field. Furthermore, this behaviour appears to be a controllable parameter that makes these systems suitable for the development of magnetic devices.

As the single nanomagnets, the $2 \mathrm{D}$ arrays also exhibit a demagnetized state as a result of the existence of two different switching fields. This behaviour allow us to select the relative magnetic state of the two layers, either parallel or antiparallel. Starting from a magnetic saturated state and applying an increasing field in opposite direction, the magnetization in one of the two layers will reverse when the applied field gets larger than the first switching field. The magnetization in the two layers is now antiparallel and the global trilayer structure is in a demagnetized state. A further saturating field will reverse the other layer leading to a parallel configuration (magnetized state). This property would have several technological applications when nanostructures in a completely demagnetized state is mandatory.

As an example of the potential applications of these systems, we have chosen the case of two particular FeCo trilayer arrays, each of them with different lateral spacing $s_{2}$ and fabricated with magnetic layers with different anisotropies (Fig. 6).

If the final lithographed sample is built up with an array with two different lateral spacings, it would be possible to obtain four separated switching field values that lead to three different magnetic states. We have lithographed the samples with a very large active area, similar to the one needed in actual devices. This is possible even for the case shown, where the two $s_{2}$ values are as similar as $160 \mathrm{~nm}$ and $180 \mathrm{~nm}$. As can be seen in the figure, the jumps take place at $\mathrm{H}_{s w}^{A 1}=-92 \pm 2 \mathrm{Oe}, \mathrm{H}_{s w}^{A 2}=262 \pm 2 \mathrm{Oe}, \mathrm{H}_{s w}^{B 1}=142 \pm 2$ Oe and $\mathrm{H}_{s w}^{B 2}=345 \pm 2$ Oe. The distance between the different switching field values are 234 Oe, 120 Oe and 83 Oe respectively and so the three magnetic signatures are separated enough to be technologically applied in multi-signatured magnetic tags for security and encoding systems [9]. 
Tailoring the magnetization states in 2-D arrays of multiresponse ferromagnetic nanomagnets 9
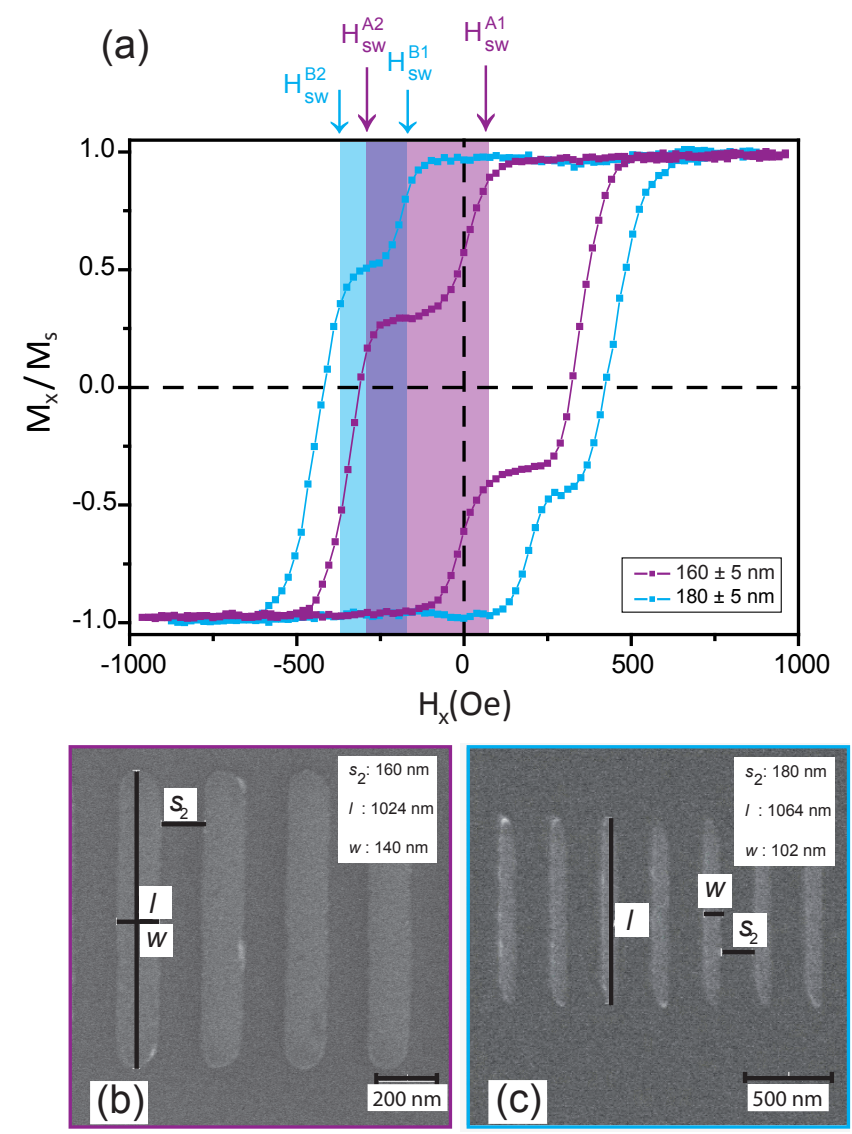

Figure 6. Example of the magnetic behaviour of two different arrays with different lateral spacing $s_{2}$ and fabricated with magnetic layers with different anisotropies. The superimposition of the two dual hysteresis loops (panel (a)) established three welldefined magnetic regions. TEM images of the two 2-D arrays are shown in panels (b) and (c).

\subsection{Micromagnetic simulations of a 2-D array of nanomagnets}

To understand the origin of the different switching fields observed in Fig. 4 we have performed micromagnetic simulations that provide detailed information about the dependence of magnetic behavior on material parameters and geometry [18]. Although there are many calculations done for micrometer sized rectangular structures [19, 20], one can expect that further reduction of the size to the nanometer scale, together with an increase of the aspect ratio, can lead to a different and/or more complex energy landscape.

The system can be well described using a model of interacting nanomagnets. Our main goal is to understand the existing stable states, the mechanisms of magnetization reversal and the influence of the aspect ratio. Notice that, in the past, simulations have been mainly applied to single magnetic structures, neglecting the interaction between adjacent elements in the array or dipolar coupled pairs [21, 22]. 
Tailoring the magnetization states in 2-D arrays of multiresponse ferromagnetic nanomagnets 10

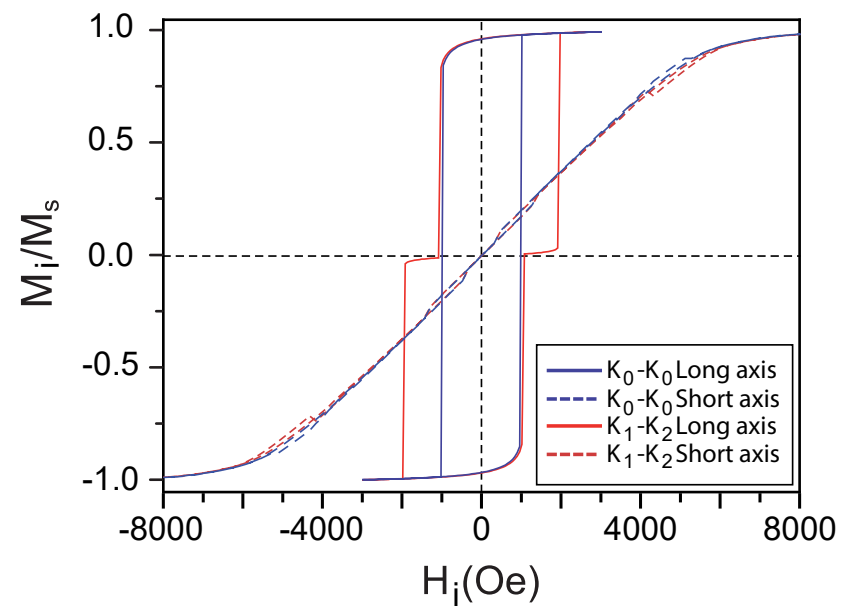

Figure 7. Effect of crystalline anisotropy on magnetization reversal in isolated trilayer nanomagnets with the same layer thicknesses $\left(t_{1}=t_{2}=20 \mathrm{~nm}, d=10 \mathrm{~nm}\right)$. The simulated hysteresis loops are shown for applied magnetic fields parallel to the short and the long axis. Two different switching fields appear for nanomagnets with different layer anisotropies (red) $\left(\mathrm{K}_{1}=1.110^{5} \mathrm{erg} / \mathrm{cm}^{3}\right.$ and $\left.K_{2}=6.710^{4} \mathrm{erg} / \mathrm{cm}^{3}\right)$ while one single jump is found when the anisotropies of the two layers are the same (blue) $\left(\mathrm{K}_{0}=0\right)$.

First, we have studied the effect of breaking the symmetry between the two magnetic layers by inducing different magnetic anisotropies in the two magnetic layers. Figure 7 reveals the difference in the hysteresis loops along the long axis between isolated trilayer nanomagnets that are geometrically identical but with different anisotropies. Simulations performed with differences in anisotropies as small as $20 \mathrm{erg} / \mathrm{cm}^{3}$ appear enough to break the symmetry and provokes the appearance a dual hysteresis loop, in agreement with the experimental data obtained for the fabricated 2D-arrays. Only a single jump is observed for nanomagnets with the same anisotropies in the magnetic layers.

To discern the magnetic coupling of the layers, we have analyzed the magnetization reversal for both, the long and the short axis, with the aspect ratio changing from 1:1 to 10:1. In Fig. 8 we plot the results obtained for the 10:1 case. Along the long axis, there is no dependence with the aspect ratio and the magnetization reversal always takes place through vortex nucleation and further propagation (not shown). The magnetization direction changes progressively and there is no change in the main magnetic state.

But along the long axis the situation is different. As it is observed in the experiments, in some occasions the hysteresis loop is dual, while others present only a single jump. To envisage the physical origin, we have drawn the magnetization distribution on the two magnetic layers on several points of the hysteresis loops. In the points marked with letter " $b$ " the magnetization along the short dimension of each magnetic layer is out of the long axis of the nanostructure (green and yellow areas in panels (b) and (c) of Fig. 8). In "c" points the yellow areas grow and invert 

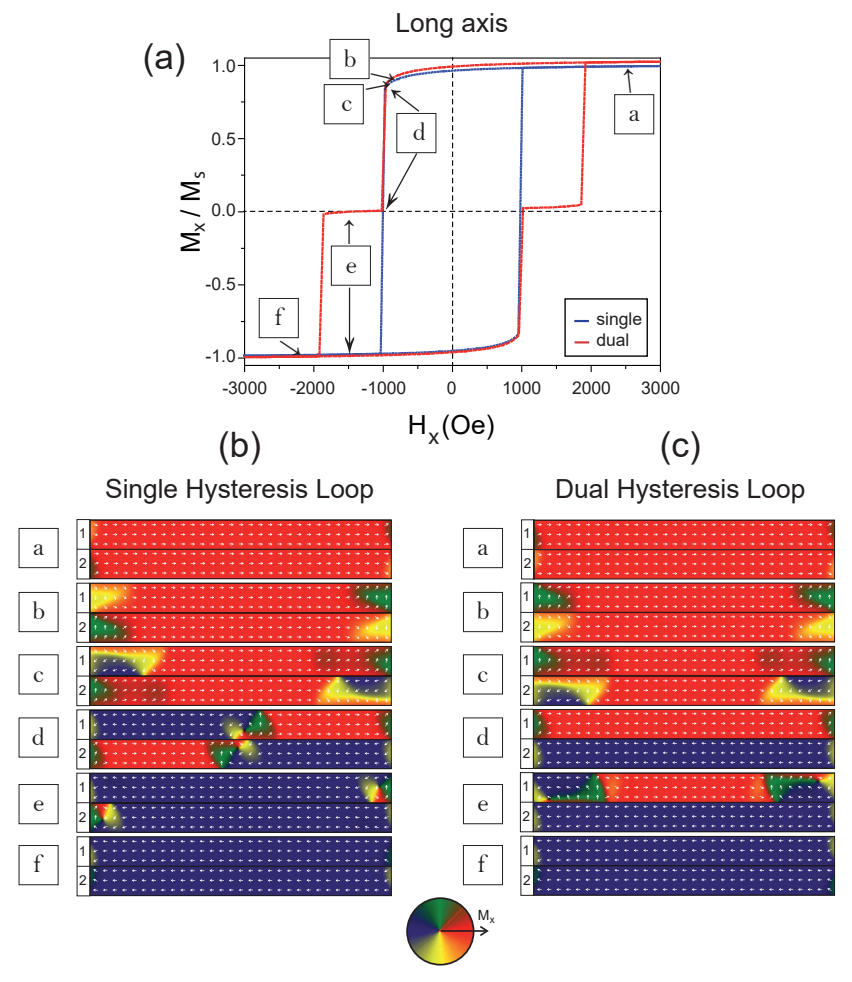

Figure 8. (s) Simulated hysteresis loops corresponding to the magnetization reversal of the nanomagnets. Low panels show the top view of the magnetization distribution of the two magnetic layers that compose the nanomagnets. The maps are extracted from the points of the hysteresis loops indicated in (a) for the single loop (b) and the dual loop (c).

their magnetization, exhibiting blue zones. They evolve differently from the areas with opposite direction (green areas), that shrink out. When these yellow areas belong to the same layer, this layer inverts the magnetization first, giving rise to two jumps in the hysteresis loop.

But although the knowledge of isolated individual nanomagnets is mandatory, real applications emerge from 2-D arrays. Fig. 9 shows the simulated hysteresis loops in 2-D arrays of trilayer FeCo nanomagnets. In an array, the relevant parameters are the spacing between adjacent nanomagnets, $s_{1}$ and $s_{2}$ (Fig. 1). Parallel to what we made in the experiments, we kept the distance between elements along the long axis fixed $\left(s_{1}=1000 \mathrm{~nm}\right)$ and we vary the separation along the short axis $\left(s_{2}\right)$. The length, the width and the thickness of the nanoelements that form the array were kept always the same ( $l=1000 \mathrm{~nm}, w=100 \mathrm{~nm}, d=10 \mathrm{~nm}$ and $t_{1}=t_{2}=20 \mathrm{~nm}$ respectively).

When the magnetic field is applied along the long axis the second switching field increases with the $s_{2}$ spacing. For the largest spacings the second switching fields become very similar and tend to those found for single nanomagnets. This is in agreement with the results found in the fabricated 2-D array which show that there is a range for $s_{2}$ values that allows us tuning the array's response in an external magnetic field. 
Tailoring the magnetization states in 2-D arrays of multiresponse ferromagnetic nanomagnets 12
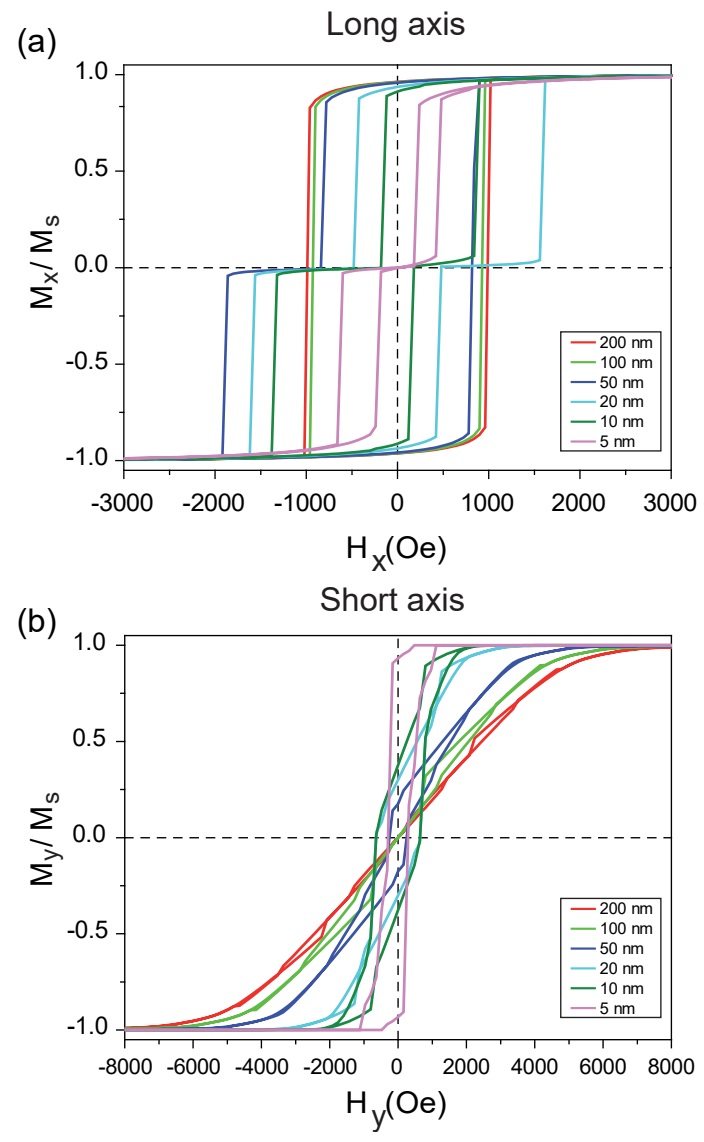

Figure 9. Calculated hysteresis loops along the (a) long and (b) short axis for 2-D arrays of trilayer nanomagnets varying $s_{2}$ and keeping $s_{1}=1000 \mathrm{~nm}$ fixed.

Unlike what we observed in the experimental values, simulations report a decrease in the first switching field when the $s_{2}$ spacing is reduced. This results indicate that experimentally, the first switching does not take place in all the nanomagnets at the same time as it is the case while using PBC's in the simulations. This restriction, together with the absence of thermal considerations in the simulated hysteresis loops, is the reason why the experimentally measured switching fields are smaller than those obtained in the simulations [23].

The two different magnetization reversal modes are also shown in Fig. 9. The single mode hysteresis loop corresponds to the nucleation of domain walls in the two layers at the same time. In the case of a single nanomagnet the coupling between the layers tends to nucleate similar structures in the two layers and therefore reverse magnetization occurs at the same field due to the fact that both layers are geometrically and magnetically identical. This trend is observed in arrays with a large spacing as it is shown for a spacings of $s_{2}=100$ and $200 \mathrm{~nm}$. So, we assigned the two mode hysteresis loop to the nucleation of domain walls first in one of the two layers giving rise to the first switching field and later, for a larger field, in the other layer leading to total magnetization reversal of the nanomagnet. In isolated nanomagnets, each layer reacts 
Tailoring the magnetization states in 2-D arrays of multiresponse ferromagnetic nanomagnets 13
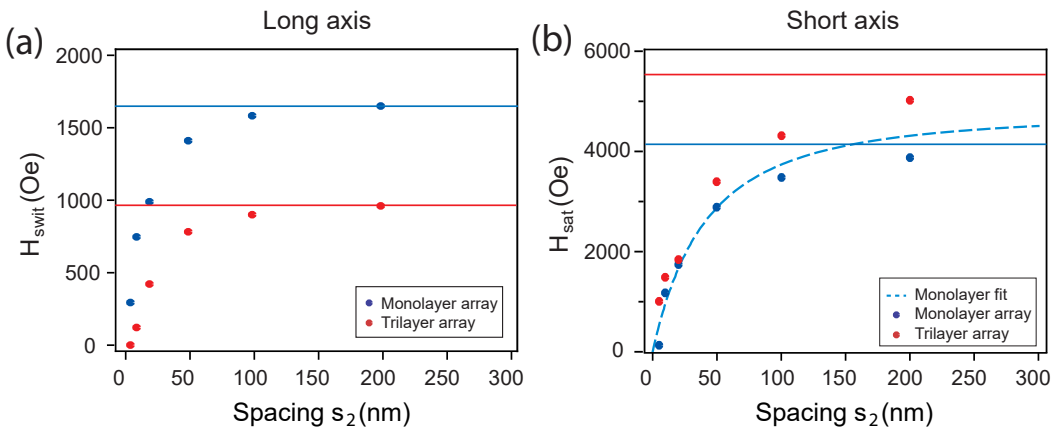

Figure 10. Dependence of the second switching field along the long axis (a) and saturation field along the short axis (b) with the spacing between adjacent nanomagnets. The red (blue) points have been obtained from micromagnetic simulations for a 2-D array of trilayer (monolayer) nanomagnets. The solid red (blue) line corresponds to the values obtained for isolated trilayer (monolayer) nanomagnets. The dashed blue line is the result of the application of the Pant's model (see text for details).

at a different magnetic field, so one layer can be reversed before the other. Once the first layer is reversed, the other requires a larger field being the larger the spacing the larger the magnetic field.

B. B. Pant proposed a simple model for calculating the saturation fields in parallel arrays of monolayer magnetic stripes $(w \gg l)$ [24]. This model was further developed by Adeyeye et al [25]. Within this model, the saturation field $(H)$ can be described as:

$$
H=M_{s} \cdot \frac{t}{l} \cdot \alpha(k)
$$

being $\alpha(k)$ a geometrical parameter defined as:

$$
\alpha(k)=\frac{2 k}{1+2 k}+\frac{k}{2(1+k)^{2}} \cdot\left(\frac{\pi^{2}}{2}-4\right)
$$

where $M_{s}$ is the saturation magnetization, $k=s_{2} / l$ is a geometrical factor, and $t$ and $l$ are layer thickness and length respectively. Fig. 10 shows the evolution of the second switching field and the saturation field as a function of $s_{2}$. As a comparison, the Figure includes the results found for arrays of nanomagnets with one magnetic layer (monolayer).

When the magnetic field is applied along the short axis, the Adeyeye's model fits very well for arrays of monolayer nanomagnets. This is expected, as in this case and this direction, the simulated array is really a 2-D array and fulfill the conditions of the Pant's model. The array behaves as a single isolated nanomagnet when the spacing $s_{2}$ is comparable to the nanomagnet length $\left(s_{2} \geq 100 \mathrm{~nm}\right)$, and so, the value for $\mathrm{H}_{\text {sat }}$ is very close to the value of isolated nanomagnet (Fig. 10a).

However, for trilayer nanomagnet arrays, the system no longer can be considered similar to a 2-D array of stripes, but as a 3-D array. Despite this, the saturation field fits well for spacings $s_{2}<20 \mathrm{~nm}$, even though Pant's model was developed for 
ribbons with infinite length and does not consider the perpendicular interaction along $\mathrm{z}$ axis, ( $d$ spacing). For these values of $s_{2}$, the magnetostatic coupling between layers in neighbor elements dominates and magnetization reversal takes place by domain wall nucleation. When $s_{2}$ increases, this coupling becomes less and less relevant with a consequent increase in the saturation field. Addionally, the experimental values for both the coercivity and the saturation fields are reduced by nearly $50 \%$ with respect to the ones found in the simulations.

Reducing the distance between adjacent elements in the array notably alters the magnetization response [26]. With the smaller calculated spacing $\left(s_{2}=5 \mathrm{~nm}\right)$, the distance between elements in the array is smaller than the distance between magnetic layers in a single element and domain walls appear, which lead to larger jumps in the loop. The y-axis has moved from a hard axis behaviour to an easy axis. For intermediate spacings, reversal magnetization takes place through magnetization rotation with two jumps in the loop due to the reversal magnetization at different magnetic fields.

When the magnetic field is applied parallel to the long axis (Fig. 10b), the condition $l>w$ is no longer met. Again, the general trend is similar in mono and trilayers, approaching both the limit corresponding to the isolated element. In this direction, the saturation field of the trilayer array is very low, giving rise to a strong magnetic sensitivity of the system. Because of the strong dependence of the saturation and the switching fields with the $s_{2}$ spacing along both axes, controlling $s_{2}$ is a simple way to tailor the saturation field as it has been confirmed in the experiments for the fabricated arrays.

\section{Conclusions}

We have analyzed the magnetic properties of 2-D arrays of FeCo trilayer nanomagnets, both experimentally and by micromagnetic simulations. We have fabricated 2-D arrays of interacting trilayer nanomagnets, grown with different magnetocrystalline anisotropies. These kind of structures have a well-defined multiresponse magnetic signature that can be tuned by carefully choosing not only the properties of the isolated nanomagnets (magnetic layers and/or aspect ratio) but the geometric dimensions of the arrays. The fabricated arrays clearly show two jumps for lateral spacings between 100 $\mathrm{nm}$ and $500 \mathrm{~nm}$.

At low spacing values, micromagnetic simulations reveal that magnetization reversal mechanism change from coherent rotation to domain wall nucleation. The saturating fields along the short axis are notably reduced. Along the long axis, magnetization reversal has two jumps corresponding to the reversal of each layer, which can be selected in a wide range with the array spacing and nanomagnet thicknesses. Very small differences in anisotropy between the magnetic layers promote the two switching fields reversal mode.

Therefore, our results prove that the lateral spacing $s_{2}$ is a simple way to tailor

the saturation and switching fields. We deem that these tailoring capabilities for the 
Tailoring the magnetization states in 2-D arrays of multiresponse ferromagnetic nanomagnets 15

magnetostatic coupling between magnetic nanoelements are potentially interesting for the development of new magnetic tags.

\section{Acknowledgments}

This work was funded by MINECO MAT2014-52477-C05-2-P, MAT2014-52477-C052-PC01-2-P, MAT2013-48009-C4-1-P and PRI-PIBUS-2011-1182. Manuel Abuín acknowledges the UCM Campus of International Excellence (PICATA Program) for PhD fellowship.

\section{References}

[1] Stamps R L, Breitkreutz S, Akerman J, Chumak A V, Otani Y, Bauer G E W, Thiele J U, Bowen M, Majetich S a, Kläui M, Prejbeanu I L, Dieny B, Dempsey N M and Hillebrands B $2014 \mathrm{~J}$. Phys. D: Appl. Phys. 47333001

[2] Lavrijsen R, Lee J H, Fernández-Pacheco A, Petit D C M C, Mansell R and Cowburn R P 2013 Nature $493647-50$

[3] Imre A, Csaba G, Ji L, Orlov a, Bernstein G H and Porod W 2006 Science 311 205-208

[4] Allwood D A, Xiong G, Faulkner C C, Atkinson D, Petit D and Cowburn R P 2005 Science 309 $1688-1692$

[5] Heyderman L J and Stamps R L 2013 J. Phys.: Condens. Mat. 25363201

[6] Yu W, Keatley P S, Gangmei P, Marcham M K, Loughran T H J, Hicken R J, Cavill S a, van der Laan G, Childress J R and Katine J a 2015 Phys. Rev. B 91 1-10

[7] Leulmi S, Joisten H, Dietsch T, Iss C, Morcrette M, Auffret S, Sabon P and Dieny B 2013 Appl. Phys. Lett. 103132412

[8] Zabow G, Dodd S, Moreland J and Koretsky A 2008 Nature 453 1058-63

[9] Love D M, Vyas K N, Fernández-Pacheco A, Llandro J, Palfreyman J J, Mitrelias T and Barnes C H W 2015 RSC Adv. 5 10211-10218

[10] Abuín M, Pérez L, Mascaraque A and Maicas M 2014 CrystEngComm. 16 9528-9533

[11] Grimsditch M and Vavassori P 2004 J. Phys.: Condens. Mat. 16 R275-R294

[12] Horcas I, Fernandez R, Gomez-Rodriguez J, Colchero J, J G H, and Baro A 2007 Rev. Sci. Instrum. 78013705

[13] Scheinfein M 2015 http://llgmicro.home.mindspring.com 11

[14] Mathieu C, Liu H J, Buchanan K S and Inturi V R 2012 J. Appl. Phys. 111 07A306

[15] Schreiber F and Frait Z 1996 Phys. Rev. B 54 6473-6480

[16] Lopez-Diaz L, Aurelio D, Torres L, Martinez E, Hernandez-Lopez M A, Gomez J, Alejos O, Carpentieri M, Finocchio G and Consolo G 2012 J. Phys. D: Appl. Phys. 45323001

[17] Lebecki K M, Donahue M J and Gutowski M W 2008 J. Phys. D: Appl. Phys. 41175005

[18] Xie K, Zhang X, Lin W, Zhang P and Sang H 2011 Phys. Rev. B 84054460

[19] of Standards N I and Technology 2000 www.ctcms.nist.gov/ rdm/mumag.html $\mathbf{x} \mathrm{xx}$

[20] Rave W, Hubert A and Member S 2000 IEEE Trans. Magn. 36 3886-3899

[21] Cowburn R 2000 J. Phys. D: Appl. Phys. 33 R1-R16

[22] Han X, Liu R, Liu Q, Wang J, Wang T and Li F 2010 Physica B 405 1172-1175

[23] Finocchio G, Carpentieri M, Azzerboni B, Torres L, Martinez E and Lopez-Diaz L $2006 \mathrm{~J}$. Appl. Phys. 99 1-4

[24] Pant B B 1996 J. Appl. Phys. 796123

[25] Adeyeye A, Bland J, Daboo C and Hasko D 1997 Phys. Rev. B 563256

[26] Goolaup S, Adeyeye a O and Singh N 2006 J. Appl. Phys. 100114301 